\title{
The current state of oligometastatic and oligoprogressive non-small cell lung cancer
}

\author{
Vasu Tumati, Puneeth Iyengar \\ Department of Radiation Oncology, University of Texas Southwestern Medical Center, Simmons Comprehensive Cancer Center, Dallas, TX, USA \\ Contributions: (I) Conception and design: All authors; (II) Administrative support: P Iyengar; (III) Provision of study materials or patients: None; \\ (IV) Collection and assembly of data: All authors; (V) Data analysis and interpretation: All authors; (VI) Manuscript writing: All authors; (VII) Final \\ approval of manuscript: All authors. \\ Correspondence to: Puneeth Iyengar, MD PhD. Department of Radiation Oncology, University of Texas Southwestern Medical Center, Simmons \\ Comprehensive Cancer Center, Dallas, TX, USA. Email: Puneeth.Iyengar@utsouthwestern.edu.
}

\begin{abstract}
Oligometastatic disease is defined as an intermediate state between localized and widespread metastatic disease. Given that in the oligometastatic state gross tumors represent the full extent of disease, there may be a role for curative local therapy despite metastatic disease. As nearly $60 \%$ of patients with nonsmall cell lung cancer (NSCLC) present with metastatic disease and another $45 \%$ of patients with initially localized disease will ultimately develop distant metastases, NSCLC represents a prime disease for aggressive intervention. In this review, the definition, prognostic factors, patient selection, rationale and evidence for treatment of oligoprogressive and oligometastatic NSCLC is discussed, including recent prospective trials and future directions.
\end{abstract}

Keywords: Non-small cell lung cancer (NSCLC); stereotactic ablative radiotherapy (SABR); stereotactic body radiation therapy (SBRT); oligometastases; oligoprogressive; metastasectomy

Submitted Oct 11, 2017. Accepted for publication Jul 04, 2018.

doi: $10.21037 /$ jtd.2018.07.19

View this article at: http://dx.doi.org/10.21037/jtd.2018.07.19

\section{Introduction}

Lung cancer is the second most commonly diagnosed cancer but the leading cause of cancer related mortality with nearly 156,000 deaths per year; of those newly diagnosed lung cancer cases approximately $85 \%$ are non-small cell lung cancer (NSCLC) $(1,2)$. Despite advances in screening nearly half of newly diagnosed NSCLC will be metastatic at presentation (3). Furthermore, despite aggressive treatment with concurrent chemotherapy and radiation followed by adjuvant chemotherapy nearly $45 \%$ of patients with locally advanced NSCLC will develop metachronous metastasis (4). Metastatic NSCLC represents the greatest contributor to cancer related mortality and thus significant research has been directed into the diagnosis and treatment of metastatic NSCLC.

Traditionally, solitary and limited metastatic NSCLC was considered a widespread and thus incurable disease.
However, investigators have begun to challenge the concept of incurable metastatic NSCLC. The principle of oligometastases was well popularized in 1995 by Hellman and Weichselbaum who hypothesized that metastatic disease occurs in a step-wise manner, initially with limited metastases followed by progression to widespread disease. Early on, metastases may be limited in number and location based on interaction of tumor cells with target organs in a "seed and soil" pattern $(5,6)$. With improvements in imaging, including positron emission tomography/ computed tomography (PET/CT) and magnetic resonance imaging (MRI), identification of isolated metastatic deposits is accomplished with higher sensitivity and specificity. A significantly greater proportion of patients may be identified early in the metastatic spectrum and offered potentially curative local treatment, creating a new paradigm in the management of limited volume metastatic NSCLC. 


\section{Defining the oligometastatic state}

While aggressive local therapy in limited metastatic sarcoma and colorectal carcinoma has been shown to lead to durable control of disease, the data supporting a true population of limited metastatic NSCLC remains limited. A meta-analysis of 49 publications, largely retrospective, including over 2,000 patients with oligometastatic NSCLC, defined as five or fewer sites of disease, was recently performed by Ashworth et al. Of note, 60\% of the studies included patients with brain only metastases. The analysis, which included patients who received aggressive local therapy to sites of metastatic disease, found a total median overall survival of 19 months and a 5 -year overall survival of $23.3 \%$ (7). The meta-analysis revealed a modest subset of patients with long-erm disease free survival and thus the existence of a true oligometastatic state. However, the studies included had highly heterogenous outcomes also suggesting that there are shortcomings in identifying patients with oligometastatic disease. A follow up individual patient data meta-analysis was performed to further identify patients that would benefit from aggressive local therapy of metastatic sites. The second meta-analysis included a total of 757 patients with five or fewer sites of metastatic disease with controlled primary sites of disease. All patients received ablative therapy to all sites of metastatic disease. The cohort of patients included had a median overall survival of 26 months, a 5 -year overall survival $29.4 \%$, and an 8 -year overall survival of $23.4 \%$. Analysis of prognostic factors coupled with recursive partitioning analysis revealed three risk groups. Those patients with metachronous development metastatic disease were considered low risk for additional metastatic disease and thus most likely to benefit from local therapy. Intermediate risk patients were those with synchronous metastatic disease but were node negative on presentation. Those patients classified as intermediate risk had 5 -year overall survivals of $36.2 \%$ and $29.2 \%$ in the training and validation sets respectively. Lastly, patients classified as high risk (synchronous metastasis and $\geq \mathrm{N} 1$ ) had 5 -year overall survivals of $13.8 \%$ and $12.1 \%$ (8). Ultimately both of the meta-analyses reveal a subset of patients who have truly oligometastatic disease and can benefit from ablative local therapy though even in those patients considered high risk for additional disease greater than $10 \%$ can benefit from aggressive local therapy.

\section{Surgery for oligometastatic disease}

Traditionally definitive management of metastatic sites consists of radical resection with or without systemic therapy. The strongest evidence for surgical management of metastatic disease is for patients with limited brain metastases. The randomized trial of whole brain radiotherapy versus surgical resection followed by whole brain radiotherapy by Patchell et al., in which $77 \%$ of patients had NSCLC, included patients with a solitary brain metastasis. The study revealed both a local control and overall survival benefit with the addition of local therapy (9). A retrospective study from Memorial Sloan Kettering Cancer Center evaluating the role of surgical resection of solitary NSCLC lung metastases revealed a 7\% 10-year overall survival (10). Taken together these studies suggest there is a benefit to aggressive local therapy in the treatment of brain metastases. While the aforementioned studies evaluated the benefit of surgical resection on only brain metastases they did not evaluate the importance of treatment of the primary site of disease. A study performed by Bonnette et al. evaluated the role of surgical resection of both the primary and synchronous brain metastases. The study, which included 103 patients, found a 1-year overall survival of $56 \%$, 2-year overall survival of $28 \%$, and an $11 \%$ overall survival at 5 years (11). An additional retrospective study performed by Collaud et al. reported on 29 patients who underwent resection of the north the primary and sites of metastatic disease. Of the 28 included in the study 19 had brain metastases, 8 lung metastases, and 2 and adrenal metastases. The 1 - and 5 -year overall survivals were $65 \%$ and $36 \%$ respectively with a median OS of 20.5 months (12).

The surgical paradigm has been further extended to extracranial metastatic sites. As the adrenal glands are a common site for metastases several studies have examined the role of adrenalectomy for patients with limited metastatic NSCLC. In the largest retrospective series, Porte et al. examined outcomes of 43 patients with either synchronous ( $\mathrm{n}=32)$ or metachronous $(\mathrm{n}=11)$ solitary adrenal metastases treated with surgery from a multi-institutional retrospective cohort. The 4-year overall survival was $11 \%$ with 3 patients surviving beyond 5 years. Interestingly, no difference in overall survival was noted between patients with synchronous or metachronous metastases (13). Several smaller retrospective series have also confirmed the findings of Porte and colleagues (14-16). The study by Congedo and colleagues further extended the surgical paradigm to other disease sites. In a mixed cohort of 53 patients with brain $(n=39)$, adrenal $(n=7)$, bone $(n=3)$, vertebra $(n=3)$, and liver $(n=1)$ metastases who underwent surgical resection the 
long-term outcomes were examined. Overall survival was $73.1 \%$ at 1 year and $24 \%$ and 5 years with a median overall survival of 19 months (17).

Given the strong suggestion of a benefit for surgical resection of metastatic disease several prospective trials have been performed. In a prospective phase II trial performed by Downey et al. 23 patients who were treated with induction chemotherapy with mitomycin, vinblastine, and cisplatin followed by resection of all sites of disease and consolidation vinblastine and cisplatin. Of note, patients with solitary brain metastases had craniotomy prior to resection. It is important to note that only 12 patients completed induction chemotherapy. However, the median overall survival was reported to be 11 months with two patients surviving to 5 years (18). In an additional trial performed by De Ruysscher and colleagues patients with $<5$ sites of metastatic disease were enrolled to a prospective phase II trial of local therapy. In the aforementioned study both surgical and radiotherapy was allowed for the treatment of metastatic sites. A total of 44 patients were enrolled but only 39 were evaluable. Of note, despite allowing for up to 5 sites of disease $87 \%$ of patients had a solitary metastatic site. Furthermore, $95 \%$ of patients received chemotherapy as part of their treatment regimen. The 1 and 3-year overall survivals were $56.4 \%$ and $17.5 \%$ respectively (19). Taken together these retrospective and limited prospective trials suggest a role for resection of all sites of gross disease in patients with limited metastatic NSCLC.

\section{Radiation therapy for oligometastatic disease}

While surgical therapy has shown promise for the treatment of oligometastatic disease, surgery is often not feasible for all sites of disease and only select patients are good surgical candidates. Furthermore, surgical treatment can often require long recoveries periods which can delay initiation or re-initiation of chemotherapy. With the invention of highly conformal radiation therapy including stereotactic ablative radiotherapy (SABR), radiation can now be used to quickly, safely, with minimal interruptions in systemic therapy in numerous disease sites (20-25). An initial retrospective analysis from the University of Chicago examined patients with oligometastatic NSCLC treated with stereotactic body radiation therapy (SBRT) to 1 to 5 sites of metastatic or primary disease following systemic therapy. Patients were treated to all known sites of active disease at a minimum of two weeks following first line systemic therapy. The most commonly used fractionation schedule was $50 \mathrm{~Gy}$ in 10 fractions. A total of 62 lesions were treated in 25 patients with a median of two lesions per patient. With a median follow-up of 14 months, the median progression free survival and overall survival was 7.6 and 22.7 months respectively. Progressive disease was identified in 52\% of patients following initial first-line chemotherapy with patients failing to have a response to therapy having worse progression free and overall survival (26). An additional retrospective study from the University of Rochester examined patients with up to five sites of oligometastatic disease of any histology (19\% NSCLC) were treated to all sites of disease with SBRT. There were 121 patients enrolled prospectively with $74 \%$ treated to 50 Gy in five fractions. The 6-year overall survival for all patients was $20 \%$. Patients who achieved a response or stable disease to initial systemic therapy prior to SBRT showed significantly higher rates of overall survival and freedom from distant metastases compared to those with progressive disease following initial systemic therapy (27). In an early prospective study of radiotherapy, the University of Chicago performed a single arm prospective dose escalation study of patients with 1-5 sites of disease. Of note, however, patients with all histologies were included and only $18 \%$ of the 61 enrolled patients had metastatic NSCLC. In the University of Chicago trial dose was escalated from 24 to 42 Gy in 3 fractions. The trial revealed a median PFS of 5.1 months with a median overall survival of approximately 2 years. While local control in the study by Salama and colleagues was low, with a 2 -year local control of $53 \%$, many patients did not receive ablative doses of radiotherapy due the dose escalation design of the trial (28).

Until recently only the aforementioned retrospective series provided evidence for the use of locally ablative radiotherapy in the treatment of limited metastatic NSCLC. Despite the lack of evidence, a significant number of practicing radiation oncologists utilized SABR in the treatment of metastatic patients. However, there have been multiple recent prospective trials elucidating the role of locally ablative therapy in limited metastatic NSCLC. In a recently published randomized phase II trial Gomez et al. investigated the role of local therapy for patients with three or fewer sites of disease after induction chemotherapy; patients with EGFR and ALK mutations were allowed on the study. Patients were randomized in a 1:1 manner to local consolidative therapy (either SBRT, surgery, or chemoradiation) with or without maintenance chemotherapy or maintenance therapy alone. Of note, observation alone was an allowed maintenance therapy. 
The trial was stopped early at an interim analysis after a total of 74 patients were enrolled. The median progression free survival was 11.9 months in the consolidative therapy arm and 3.9 months in the maintenance only arm. An interesting finding of the Gomez study was that the time to development of a new site of disease was prolonged in the consolidative arm when compared to the maintenance alone arm (29). In addition to the study by Gomez and colleagues, we performed a randomized phase II trial of maintenance chemotherapy alone vs. SBRT and maintenance chemotherapy. In our trial patients with six or fewer sites of disease after response or stable disease after induction chemotherapy were randomized to SBRT (hypofractionated radiotherapy to the primary and mediastinum was allowed) followed by maintenance chemotherapy or maintenance chemotherapy alone. Patients with EGFR and ALK mutations were not allowed on trial. The progression free survival was 9.7 months in the SBRT plus maintenance chemotherapy arm and 3.5 months in the maintenance alone arm. None of the patients who progressed in the SBRT plus maintenance chemotherapy arm progressed at an initial site of disease and treatment dramatically shifted the expected patterns of failure (30). While the recently published trials confirmed a progression free survival benefit for local therapy in limited metastatic NSCLC the more important question of the effect on overall survival remains unanswered. There are currently two trials underway SABR-COMET (NCT01446744) and NRGLU-002 (NCT03137771) which are powered to detect an overall survival benefit and will definitely elucidate the role of locally ablative therapy in the treatment of limited metastatic NSCLC.

\section{Oligoprogressive NSCLC}

Many studies have focused on first line treatment of patients with limited synchronous or metachronous metastatic disease; however, there exists a separate population of metastatic patients' worth discussion. Recent genomic studies have revealed that there is distinct clonal evolution at each site of metastatic disease such that individual sites may obtain treatment resistance or increased metastatic potential independent of the primary site of disease or even other metastatic sites. These patients with progression limited to only a few sites of disease may warrant treatment with locally ablative therapy. A retrospective study by Gan et al. examined the role of local therapy in patients with ALK mutations who progressed on crizotinib therapy.
A total of 33 patients with $1-3$ sites of extracranial oligoprogressive disease were included. Of the 33 patients included 18 had extracranial progression, and of those 18, 14 received consolidative local therapy. For those patients receiving local therapy the median progression free survival was 14 months compared to 7.2 months for those without local therapy (31). A similar retrospective study was performed by at Memorial Sloan Kettering Cancer Center in patients with EGFR mutated NSCLC. In the study by Yu et al. patients who had acquired resistance to traditional EGFR inhibitors such as erlotinib or gefitinib were treated with locally ablative therapy. Of the 142 patients found to have progression on a tyrosine kinase inhibitor (TKI) only 18 were treated with locally ablative therapy to a non-CNS site of progression. In the cohort of 18 patients' median progression free survival was 10 months with a median overall survival of 41 months (32). A study by Weickhardt and colleagues evaluated the benefits of aggressive local ablative therapy for patients with central nervous system and/or limited systemic disease progression. They also described continuation of treatment with crizotinib or erlotinib in patients with metastatic $A L K$ gene rearrangement $(\mathrm{n}=38)$ or EGFR mutation $(\mathrm{n}=27)$ NSCLC, respectively. In their study, the median progression free survival was 9.0 months for ALK patients and 13.8 months for EGFR mutant patients. Twenty-five patients (49\%) who progressed received local therapy (15 ALK, 10 EGFR) and continuation of the same targeted therapy. In those patients in whom local therapy was used, 19 of 25 patients had a subsequent progression with median subsequent PFS of 6.2 months (33). Lastly, a prospective single arm phase II study investigating the role of local therapy was recently reported by our group. In the study patients with < six sites of extra-cranial progression who had progressed on at least one line of chemotherapy were treated with SBRT and maintenance erlotinib. Of note, the study was performed prior to the indication for erlotinib therapy being limited to patients with known EGFR mutations and thus the mutation status was not checked on all patients enrolled. Thirteen of the 24 patients enrolled on trial had EGFR mutation status analyzed but all were wild type. SBRT was delivered to a range of extracranial sites, and to two or more sites in $62.5 \%$ of patients. A change in the pattern of relapse was noted, with a shift in failure from treated sites of known disease to new sites of distant failure. By treating new sites of progression with SBRT, several patients in this study were able to remain on erlotinib for additional periods of 6 to 9 months. Toxicities were limited, including two grade 3 
toxicities, four grade 4 toxicities (one possibly from SBRT, and three definitely related to erlotinib), and one grade 5 toxicity (death) possibly related to SBRT. The median PFS and OS for patients in the study were 14.7 and 20.4 months, respectively (34). While the aforementioned retrospective and single arm prospective trials compare favorably with historic controls the lack of appropriate controls confounds the magnitude of benefit for treatment of oligoprogressive disease. The currently enrolling STOP (NCT02756793) and HALT (NCT03256981) trials are randomized trials evaluating the role of locally ablative therapy on progression free survival in patients with oligoprogressive NSCLC.

\section{Immunotherapy and future directions}

Therapy for NSCLC has seen significant advances within the last few years. The standard of care for patients with metastatic NSCLC has consisted of platinum-based chemotherapy followed by maintenance chemotherapy (35-41). However, for those patients with PD-1 expression $>50 \%$ there is an overall survival benefit associated with the use of pembrolizumab over traditional platinum chemotherapy (42). In addition, in those patients who have progressed after first line chemotherapy there is an overall survival benefit using nivolumab over single agent docetaxel (43). Several retrospective series and case reports have shown significant out of field, or abscopal, effects with the use of radiation therapy in NSCLC though less frequently than with other solid malignancies (44-46). A single arm single institution prospective trial of 35 Gy in 10 fractions delivered with concurrently with granulocytemacrophage colony-stimulating factor (GM-CSF) revealed nearly $30 \%$ of patients had an abscopal response in untreated lesions (47). These early findings strongly support the use of ablative radiotherapy in patients with metastatic NSCLC. However, there are still many concerns with combined immunotherapy and radiation: (I) there are no predictive clinical or biologic parameters that can accurately predict in whom abscopal effects are likely (II) the optimal dose of radiotherapy to elicit immune stimulation remains unclear (III) the proper order of therapy, sequential or concurrent, also remains unclear. Further studies are necessary to further elucidate patient selection and appropriate radiotherapy in the immunotherapy era.

As the indications for immunotherapy continue to expand the appropriate role of local therapy continues to change. The recently published PACIFIC trial showed a significant progression free survival benefit for the addition of adjuvant durvalumab after chemoradiotherapy (48). While the incidence of new lesions was reduced to $20 \%$ from $32 \%$ with the addition durvalumab this still leaves 1 in 5 patients with metachronous metastatic disease (48). However, whereas these patients were previously treated with chemotherapy alone these new lesions are ostensibly resistant to anti-PD1 therapy and thus the optimal therapy remains unclear for these patients. While many resistance mechanisms have been suggested in pre-clinical models of most interest is T-cell exhaustion via upregulation of multiple inhibitory molecules such as CTLA-4 (49). A phase II trial by Hellmann et al. investigated the role of CTLA4 and PD-1 combined blockade. A total of 78 patients were randomized to nivolumab every two weeks with either ipilimumab every 6 or 12 weeks. Treatment-related serious adverse events were reported in $12(32 \%)$ and $11(28 \%)$ patients in the 6 and 12 -week cohorts. Objective responses were achieved in $47 \%$ of patients. The combination was felt to be safe and tolerable (50). While early studies have shown promise for CTLA-4 inhibitors in the metastatic setting more recent results have been mixed. A recent study by Govindan et al. investigated the use of ipilimumab for patients with metastatic or recurrent NSCLC. Patients were randomized to carboplatin and paclitaxel with either placebo or ipilimumab. Median overall survival was 13.4 months for chemotherapy plus ipilimumab and 12.4 months for chemotherapy plus placebo; the difference was not found to be statistically significant. Median progression-free survival was 5.6 months for both groups (51). As these studies did not investigate the oligoprogressive setting or the role of radiotherapy in stimulating immune response to overcome resistance mechanisms there are significant areas that will require intensive study. At this point, the only statement that can be made is that combination therapy appears to be safe for patients with NSCLC. The use of combined blockade and radiation therapy are currently being investigated in NCT03223155 and NCT03168464. The role of CTLA-4, PDL-2 and other immunotherapies in combination with ablative radiotherapy in the post-primary PD-1 inhibition era will require rigorous additional study.

\section{Conclusions}

The understanding and treatment of metastatic NSCLC has seen significant advances within the last few years. However significant gaps in our understanding of patient selection, optimal systemic therapy, the tumor microenvironment, 
and the effects of radiotherapy on the immune system both locally and systemically still exist. Significant advances and intensive study will be required to increase disease free and overall survival in patients with limited NSCLC. Despite these limitations however there still exists a population in whom long term cure is possible and with further study these patients can reliably treated and ultimately cured with aggressive therapy.

\section{Acknowledgements}

None.

\section{Footnote}

Conflicts of Interest: The authors have no conflicts of interest to declare.

\section{References}

1. Siegel RL, Miller KD, Jemal A. Cancer Statistics, 2017. CA Cancer J Clin 2017;67:7-30.

2. Chen VW, Ruiz BA, Hsieh MC, et al. Analysis of stage and clinical/prognostic factors for lung cancer from SEER registries: AJCC staging and collaborative stage data collection system. Cancer 2014;120 Suppl 23:3781-92.

3. Rusch VW, Rice TW, Crowley J, et al. The seventh edition of the American Joint Committee on Cancer/ International Union Against Cancer Staging Manuals: the new era of data-driven revisions. J Thorac Cardiovasc Surg 2010;139:819-21.

4. Bradley JD, Paulus R, Komaki R, et al. Standard-dose versus high-dose conformal radiotherapy with concurrent and consolidation carboplatin plus paclitaxel with or without cetuximab for patients with stage IIIA or IIIB non-small-cell lung cancer (RTOG 0617): a randomised, two-by-two factorial phase 3 study. Lancet Oncol 2015;16:187-99.

5. Weichselbaum RR, Hellman S. Oligometastases revisited. Nat Rev Clin Oncol 2011;8:378-82.

6. Hellman S, Weichselbaum RR. Oligometastases. J Clin Oncol 1995;13:8-10.

7. Ashworth $\mathrm{A}$, Rodrigues $\mathrm{G}$, Boldt $\mathrm{G}$, et al. Is there an oligometastatic state in non-small cell lung cancer? A systematic review of the literature. Lung Cancer 2013;82:197-203.

8. Ashworth AB, Senan S, Palma DA, et al. An individual patient data metaanalysis of outcomes and prognostic factors after treatment of oligometastatic non-small-cell lung cancer. Clin Lung Cancer 2014;15:346-55.

9. Patchell RA, Tibbs PA, Walsh JW, et al. A randomized trial of surgery in the treatment of single metastases to the brain. N Engl J Med 1990;322:494-500.

10. Burt $M$, Wronski $M$, Arbit E, et al. Resection of brain metastases from non-small-cell lung carcinoma. Results of therapy. Memorial Sloan-Kettering Cancer Center Thoracic Surgical Staff. J Thorac Cardiovasc Surg 1992;103:399-410; discussion 410-1.

11. Bonnette P, Puyo P, Gabriel C, et al. Surgical management of non-small cell lung cancer with synchronous brain metastases. Chest 2001;119:1469-75.

12. Collaud S, Stahel R, Inci I, et al. Survival of patients treated surgically for synchronous single-organ metastatic NSCLC and advanced pathologic TN stage. Lung Cancer 2012;78:234-8.

13. Porte H, Siat J, Guibert B, et al. Resection of adrenal metastases from non-small cell lung cancer: a multicenter study. Ann Thorac Surg 2001;71:981-5.

14. Luketich JD, Burt ME. Does resection of adrenal metastases from non-small cell lung cancer improve survival? Ann Thorac Surg 1996;62:1614-6.

15. Porte HL, Roumilhac D, Graziana JP, et al. Adrenalectomy for a solitary adrenal metastasis from lung cancer. Ann Thorac Surg 1998;65:331-5.

16. Lucchi M, Dini P, Ambrogi MC, et al. Metachronous adrenal masses in resected non-small cell lung cancer patients: therapeutic implications of laparoscopic adrenalectomy. Eur J Cardiothorac Surg 2005;27:753-6.

17. Congedo MT, Cesario A, Lococo F, et al. Surgery for oligometastatic non-small cell lung cancer: longterm results from a single center experience. J Thorac Cardiovasc Surg 2012;144:444-52.

18. Downey RJ, Ng KK, Kris MG, et al. A phase II trial of chemotherapy and surgery for non-small cell lung cancer patients with a synchronous solitary metastasis. Lung Cancer 2002;38:193-7.

19. De Ruysscher D, Wanders R, van Baardwijk A, et al. Radical treatment of non-small-cell lung cancer patients with synchronous oligometastases: long-term results of a prospective phase II trial (Nct01282450). J Thorac Oncol 2012;7:1547-55.

20. Folkert MR, Bilsky MH, Tom AK, et al. Outcomes and toxicity for hypofractionated and single-fraction image-guided stereotactic radiosurgery for sarcomas metastasizing to the spine. Int J Radiat Oncol Biol Phys 2014;88:1085-91. 
21. Hannan R, Tumati V, Xie XJ, et al. Stereotactic body radiation therapy for low and intermediate risk prostate cancer-Results from a multi-institutional clinical trial. Eur J Cancer 2016;59:142-51.

22. Meyer JJ, Foster RD, Lev-Cohain N, et al. A Phase I Dose-Escalation Trial of Single-Fraction Stereotactic Radiation Therapy for Liver Metastases. Ann Surg Oncol 2016;23:218-24.

23. Rusthoven KE, Kavanagh BD, Burri SH, et al. Multiinstitutional phase I/II trial of stereotactic body radiation therapy for lung metastases. J Clin Oncol 2009;27:1579-84.

24. Rusthoven KE, Kavanagh BD, Cardenes H, et al. Multiinstitutional phase I/II trial of stereotactic body radiation therapy for liver metastases. J Clin Oncol 2009;27:1572-8.

25. Timmerman RD, Kavanagh BD, Cho LC, et al. Stereotactic body radiation therapy in multiple organ sites. J Clin Oncol 2007;25:947-52.

26. Hasselle MD, Haraf DJ, Rusthoven KE, et al. Hypofractionated image-guided radiation therapy for patients with limited volume metastatic non-small cell lung cancer. J Thorac Oncol 2012;7:376-81.

27. Milano MT, Katz AW, Zhang H, et al. Oligometastases treated with stereotactic body radiotherapy: long-term follow-up of prospective study. Int J Radiat Oncol Biol Phys 2012;83:878-86.

28. Salama JK, Hasselle MD, Chmura SJ, et al. Stereotactic body radiotherapy for multisite extracranial oligometastases: final report of a dose escalation trial in patients with 1 to 5 sites of metastatic disease. Cancer 2012;118:2962-70.

29. Gomez DR, Blumenschein GR Jr, Lee JJ, et al. Local consolidative therapy versus maintenance therapy or observation for patients with oligometastatic non-smallcell lung cancer without progression after first-line systemic therapy: a multicentre, randomised, controlled, phase 2 study. Lancet Oncol 2016;17:1672-82.

30. Iyengar P, Wardak Z, Gerber DE, et al. Consolidative radiotherapy for limited metastatic non-small-cell lung cancer: A phase 2 randomized clinical trial. JAMA Oncol 2018;4:e173501.

31. Gan GN, Weickhardt AJ, Scheier B, et al. Stereotactic radiation therapy can safely and durably control sites of extra-central nervous system oligoprogressive disease in anaplastic lymphoma kinase-positive lung cancer patients receiving crizotinib. Int J Radiat Oncol Biol Phys 2014;88:892-8.

32. Yu HA, Sima CS, Huang J, et al. Local therapy with continued EGFR tyrosine kinase inhibitor therapy as a treatment strategy in EGFR-mutant advanced lung cancers that have developed acquired resistance to EGFR tyrosine kinase inhibitors. J Thorac Oncol 2013;8:346-51.

33. Weickhardt AJ, Scheier B, Burke JM, et al. Local ablative therapy of oligoprogressive disease prolongs disease control by tyrosine kinase inhibitors in oncogeneaddicted non-small-cell lung cancer. J Thorac Oncol 2012;7:1807-14.

34. Iyengar P, Kavanagh BD, Wardak Z, et al. Phase II trial of stereotactic body radiation therapy combined with erlotinib for patients with limited but progressive metastatic non-small-cell lung cancer. J Clin Oncol 2014;32:3824-30.

35. Brodowicz T, Ciuleanu T, Crawford J, et al. Third CECOG consensus on the systemic treatment of nonsmall-cell lung cancer. Ann Oncol 2012;23:1223-9.

36. Brodowicz T, Krzakowski M, Zwitter M, et al. Cisplatin and gemcitabine first-line chemotherapy followed by maintenance gemcitabine or best supportive care in advanced non-small cell lung cancer: a phase III trial. Lung Cancer 2006;52:155-63.

37. Paz-Ares LG, de Marinis F, Dediu M, et al. PARAMOUNT: Final overall survival results of the phase III study of maintenance pemetrexed versus placebo immediately after induction treatment with pemetrexed plus cisplatin for advanced nonsquamous non-small-cell lung cancer. J Clin Oncol 2013;31:2895-902.

38. Behera M, Owonikoko TK, Chen Z, et al. Single agent maintenance therapy for advanced stage non-small cell lung cancer: a meta-analysis. Lung Cancer 2012;77:331-8.

39. Ciuleanu T, Brodowicz T, Zielinski C, et al. Maintenance pemetrexed plus best supportive care versus placebo plus best supportive care for non-small-cell lung cancer: a randomised, double-blind, phase 3 study. Lancet 2009;374:1432-40.

40. Fidias PM, Dakhil SR, Lyss AP, et al. Phase III study of immediate compared with delayed docetaxel after frontline therapy with gemcitabine plus carboplatin in advanced non-small-cell lung cancer. J Clin Oncol 2009;27:591-8.

41. Gerber DE. Maintenance therapy for advanced lung cancer: who, what, and when? J Clin Oncol 2013;31:2983-90.

42. Shaverdian N, Lisberg AE, Bornazyan K, et al. Previous radiotherapy and the clinical activity and toxicity of pembrolizumab in the treatment of non-small-cell lung cancer: a secondary analysis of the KEYNOTE-001 phase 1 trial. Lancet Oncol 2017;18:895-903. 
43. Borghaei H, Paz-Ares L, Horn L, et al. Nivolumab versus Docetaxel in Advanced Nonsquamous Non-Small-Cell Lung Cancer. N Engl J Med 2015;373:1627-39.

44. Siva S, Callahan J, MacManus MP, et al. Abscopal [corrected] effects after conventional and stereotactic lung irradiation of non-small-cell lung cancer. J Thorac Oncol 2013;8:e71-2.

45. Cong Y, Shen G, Wu S, et al. Abscopal regression following SABR for non-small-cell-lung cancer: A case report. Cancer Biol Ther 2017;18:1-3.

46. Golden EB, Demaria S, Schiff PB, et al. An abscopal response to radiation and ipilimumab in a patient with metastatic non-small cell lung cancer. Cancer Immunol Res 2013;1:365-72.

47. Golden EB, Chhabra A, Chachoua A, et al. Local radiotherapy and granulocyte-macrophage colonystimulating factor to generate abscopal responses in

Cite this article as: Tumati V, Iyengar P. The current state of oligometastatic and oligoprogressive non-small cell lung cancer. J Thorac Dis 2018;10(Suppl 21):S2537-S2544. doi: 10.21037/ jtd.2018.07.19 patients with metastatic solid tumours: a proof-of-principle trial. Lancet Oncol 2015;16:795-803.

48. Antonia SJ, Villegas A, Daniel D, et al. Durvalumab after Chemoradiotherapy in Stage III Non-Small-Cell Lung Cancer. N Engl J Med 2017;377:1919-29.

49. O'Donnell JS, Long GV, Scolyer RA, et al. Resistance to PD1/PDL1 checkpoint inhibition. Cancer Treat Rev 2017;52:71-81.

50. Hellmann MD, Rizvi NA, Goldman JW, et al. Nivolumab plus ipilimumab as first-line treatment for advanced nonsmall-cell lung cancer (CheckMate 012): results of an open-label, phase 1, multicohort study. Lancet Oncol 2017;18:31-41.

51. Govindan R, Szczesna A, Ahn MJ, et al. Phase III Trial of Ipilimumab Combined With Paclitaxel and Carboplatin in Advanced Squamous Non-Small-Cell Lung Cancer. J Clin Oncol 2017;35:3449-57. 